\title{
3D magnetotelluric inversion using a limited-memory quasi-Newton optimization
}

\author{
Dmitry Avdeev ${ }^{1}$ and Anna Avdeeva ${ }^{2}$
}

\begin{abstract}
The limited-memory quasi-Newton method with simple bounds is used to develop a novel, fully 3D magnetotelluric (MT) inversion technique. This nonlinear inversion is based on iterative minimization of a classical Tikhonov regularized penalty function. However, instead of the usual model space of $\log$ resistivities, the approach iterates in a model space with simple bounds imposed on the conductivities of the 3D target. The method requires storage proportional to $2 \times n_{c p}$ $\times N$, where $N$ is the number of conductivities to be recovered and $n_{c p}$ is the number of correction pairs (practically, only a few). These requirements are much less than those imposed by other Newton methods, which usually require storage proportional to $N \times M$ or $N \times N$, where $M$ is the number of data to be inverted. The derivatives of the penalty function are calculated using an adjoint method based on electromagnetic field reciprocity. The inversion involves all four entries of the MT impedance matrix; the $x 3 \mathrm{D}$ integral equation forwardmodeling code is used as an engine for this inversion. Convergence, performance, and accuracy of the inversion are demonstrated on synthetic numerical examples. After investigating erratic resistivities in the upper part of the model obtained for one of the examples, we conclude that the standard Tikhonov regularization is not enough to provide consistently smooth underground structures. An additional regularization helps to overcome the problem.
\end{abstract}

\section{INTRODUCTION}

Limited-memory quasi-Newton (QN) methods have become very popular tools to solve 3D electromagnetic (EM) large-scale inverse problems numerically (Newman and Boggs, 2004; Haber, 2005; Plessix and Mulder, 2008). The methods require calculating gradi- ents of the misfit only yet avoid calculating second-derivative terms. Only several pairs of so-called correction vectors are needed, dramatically diminishing storage requirements. However, the inherent disadvantage of this approach is that it can converge slowly. An effective way to accelerate the solution is to calculate the gradients using an adjoint method. A more complete review on this subject is found in Avdeev (2005).

In this paper, we apply a limited-memory QN method with simple bounds to solve the 3D magnetotelluric (MT) inverse problem. First, we describe the setting of the inverse problem as well as some key features of our implementation, referring the reader to Avdeeva and Avdeev (2006) for details. Then, we develop the theory and basic equations to calculate gradients of the misfit. We demonstrate that the calculation of gradients at a given period is equivalent to only two forward modelings and does not depend on the number of conductivities to be recovered. The mathematical details of the approach are described in greater detail in four appendices.

This is followed by a demonstration of how our inversion works practically on synthetic numerical examples. One of the examples includes an outcropping tilted conductive dike in uniform halfspace. Another example is more complex, involving a model with resistive and conductive adjacent blocks buried in a two-layered earth. Both models have been used to test other forward and inverse codes. For the adjacent blocks model, we encounter the problem that reasonable resistivity values are recovered only exactly under the cells beneath the MT sites. For these cells, it is possible to see the underground structure yet difficult to reconstruct the resistivity elsewhere. The resistivity image looks very rough, especially at the upper part of the model. Tikhonov regularization alone is not enough to solve this problem; an additional regularization must be used. We introduce this regularization and demonstrate how it improves the inversion results.

Our results are encouraging and suggest that the inversion can be applied successfully to solve realistic 3D inverse problems with real MT data.

Manuscript received by the Editor 13 July 2008; revised manuscript received 14 November 2008; published online 27 April 2009 ; corrected version published online 30 April 2009.

${ }^{1}$ Formerly Dublin Institute for Advanced Studies, Dublin, Ireland; presently Russian Academy of Sciences, Pushkov Institute of Terrestrial Magnetism, Ionosphere and Radiowave Propagation, Moscow, Russia. E-mail: davdeev@izmiran.ru.

${ }^{2}$ Formerly Dublin Institute for Advanced Studies, Dublin, Ireland; presently IFM-GEOMAR, Kiel, Germany. E-mail: aavdeeva@ ifm-geomar.de. (C) 2009 Society of Exploration Geophysicists. All rights reserved. 


\section{D MT INVERSION}

First, let us consider a 3D earth conductivity model discretized by $N$ cells, so that $\boldsymbol{\sigma}(\mathbf{r})=\sum_{k=1}^{N} \sigma_{k} \chi_{k}(\mathbf{r})$, where

$$
\chi_{k}(\mathbf{r})=\left\{\begin{array}{l}
1, \mathbf{r} \in V_{k} \\
0, \mathbf{r} \notin V_{k}
\end{array} \quad, \quad \mathbf{r}=(x, y, z)\right.
$$

and $V_{k}$ is the volume occupied by the $k$ th cell. In the frame of 3D MT inversion, conductivities $\sigma_{k}(k=1, \ldots, N)$ of the cells are sought. This problem can be viewed as a typical optimization, so that $\varphi(\boldsymbol{\sigma}, \lambda) \rightarrow \min$, with a penalty function $\varphi$ given as $\boldsymbol{\sigma}, \lambda$

$$
\varphi(\boldsymbol{\sigma}, \lambda)=\varphi_{d}(\boldsymbol{\sigma})+\lambda \varphi_{s}(\boldsymbol{\sigma})
$$

where

$$
\varphi_{d}=\frac{1}{2} \sum_{j=1}^{N_{S}} \sum_{n=1}^{N_{T}} \beta_{j n} t r\left[\overline{\mathbf{A}}_{j n}^{T} \mathbf{A}_{j n}\right]
$$

is a measure of the data misfit. Here, $\boldsymbol{\sigma}=\left(\sigma_{1}, \ldots, \sigma_{N}\right)^{T}$ is the vector consisting of the electrical conductivities of the cells, superscript $T$ means transpose, the overbar stands for the complex conjugate, $N$ is the number of the cells, $N_{S}$ is the number of MT sites, $\mathbf{r}_{j}=\left(x_{j}, y_{j}, z_{j}\right)$ where $j=1, \ldots, N_{S}$, and $N_{T}$ is the number of the frequencies $\omega_{n}$ where $n=1, \ldots, N_{T}$. The $2 \times 2$ matrices $\mathbf{A}_{j n}$ are defined as $\mathbf{A}_{j n}$ $=\mathbf{Z}_{j n}-\mathbf{D}_{j n}$, where

$$
\mathbf{Z}_{j n}=\left(\begin{array}{ll}
Z_{x x} & Z_{x y} \\
Z_{y x} & Z_{y y}
\end{array}\right)_{j n} \quad \text { and } \quad \mathbf{D}_{j n}=\left(\begin{array}{cc}
D_{x x} & D_{x y} \\
D_{y x} & D_{y y}
\end{array}\right)_{j n}
$$

are matrices of the complex-valued predicted $\mathbf{Z}\left(\mathbf{r}_{j}, \omega_{n}\right)$ and observed $\mathbf{D}\left(\mathbf{r}_{j}, \omega_{n}\right)$ impedances, respectively (see Appendix B for details). In addition,

$$
\beta_{j n}=\frac{1}{N_{S} N_{T}} \frac{2}{\varepsilon_{j n}^{2} \operatorname{tr}\left[\overline{\mathbf{D}}_{j n}^{T} \mathbf{D}_{j n}\right]}
$$

are the positive weights, where $\varepsilon_{j n}$ is the relative error of the observed impedance $\mathbf{D}_{j n}$ and $\lambda$ is the regularization parameter. The value $\operatorname{tr}[\cdot]$ means the trace of its matrix argument, defined as $\operatorname{tr}[\mathbf{B}]$ $=B_{x x}+B_{y y}$ for any

$$
\mathbf{B}=\left(\begin{array}{ll}
B_{x x} & B_{x y} \\
B_{y x} & B_{y y}
\end{array}\right)
$$

The question of why the form of equation 2 was chosen to represent a measure of the misfit is discussed in Appendix A. In addition, a more generalized form of equation 2 is considered in Appendix D.

As prescribed by the regularization theory of Tikhonov and Arsenin (1977), the penalty function $\varphi$ of equation 1 has a regularized part (a stabilizer) $\varphi_{s}(\boldsymbol{\sigma})$. This stabilizer can be chosen in different ways (see Farquharson and Oldenburg, 1998); moreover, the correct choice of $\varphi_{s}(\boldsymbol{\sigma})$ is crucial for a reliable inversion. However, this aspect of the problem is beyond the scope of this paper. Thus, we consider a conventional smoothing stabilizer given by

$$
\varphi_{s}(\boldsymbol{\sigma})=\sum_{k^{\prime}=1}^{N}\left(\sum_{k=1}^{N} W_{k^{\prime} k} \sigma_{k}\right)^{2}
$$

where the coefficients $W_{k^{\prime} k}\left(k^{\prime}, k=1, \ldots, N\right)$ represent a finite-dif- ference approximation to the Laplace operator that controls model smoothness.

When the stabilizer is used in the inversion, we encounter the additional problem of finding the optimum regularization parameter $\lambda$. In Avdeeva and Avdeev (2006), we propose an approach for finding the regularization parameter for the 1D MT inversion case. There we solve several inverse problems with a fixed value of $\lambda$, starting from the same initial guess model. For the $3 \mathrm{D}$ case, the inversion can take several days to compute, which is much too time consuming.

Therefore, for the 3D case, we choose $\lambda$ in a manner similar to that of Haber et al. (2000). A relatively large value of $\lambda$ is assigned initially and then reduced gradually. Each new problem is solved using the solution of the previous problem (i.e., the model obtained using the previous value of $\lambda$ ) as an initial guess. How to choose the initial value for the regularization parameter $\lambda$ and how fast it should be reduced at this moment depends on the experience of the user and some automatic schemes that must be developed. The so-called multiplicative regularization technique (Abubakar et al., 2008), which introduces an automated way to choose the regularization parameter adaptively, might be an example to follow.

Because the conductivities $\sigma_{k}(k=1, \ldots, N)$ must be nonnegative and realistic, it is important that the optimization problem of equations $1-3$ be subject to bounds

$$
l_{k} \leq \sigma_{k} \leq u_{k}
$$

where $l_{k}$ and $u_{k}$ are the lower and upper bounds and $l_{k} \geq 0$ ( $k$ $=1, \ldots, N)$, respectively. An alternative way to keep the conductivities positive is to consider the $\log$ conductivities $-\log \left(\sigma_{k}-l_{k}\right)$ or $\log \left(\left(\sigma_{k}-l_{k}\right) /\left(u_{k}-\sigma_{k}\right)\right)$ - as unknown parameters. After such transformations, the bounds of the model parameters extend at infinity and the constrained problem of equations $1-4$ turns nominally to an easier unconstrained problem of equations 1-3.

\section{A quasi-Newton method}

The problem given in equations 1-4 is a typical optimization problem with simple bounds (Nocedal and Wright, 1999). To solve it, we apply the limited-memory quasi-Newton method with simple bounds. Our implementation of the method is slightly different than that of Byrd et al. (1995). It is described in Avdeeva and Avdeev (2006), which applies the method to the 1D problem. However, for the $3 \mathrm{D}$ problem considered in this paper, we apply the method within a new model space $\mathbf{m}=\left(m_{1}, \ldots, m_{N}\right)^{T}$ of the new model parameters $m_{k}=\sigma_{k} / \sigma_{k}^{(0)}$, where $\sigma_{k}^{(0)}$ is the conductivity of $k$ th cell for an initialguess model. At each iteration step $l$, we find the search direction $\mathbf{p}^{(l)}$ $=\left(p_{1}^{(l)}, \ldots, p_{N}^{(l)}\right)^{T}$ as

$$
\mathbf{p}^{(l)}=-\mathbf{G}^{(l)} \nabla_{\mathbf{m}} \varphi^{(l)}
$$

where

$$
\nabla_{\mathbf{m}} \varphi^{(l)}=\left.\left(\frac{\partial \varphi}{\partial m_{1}}, \ldots, \frac{\partial \varphi}{\partial m_{N}}\right)^{T}\right|_{\sigma=\sigma^{(l)}}
$$

is the gradient vector and $\mathbf{G}^{(l)}$ is an approximation to the inverse Hessian matrix, updated at every iteration using the limited-memory Broyden-Fletcher-Goldfarb-Shanno (BFGS) formula (see Nocedal and Wright, 1999; their formula 9.5). The next iterate, $\boldsymbol{\sigma}^{(l+1)}$ $=\left(\sigma_{1}^{(l+1)}, \ldots, \sigma_{N}^{(l+1)}\right)^{T}$, is found as 


$$
\sigma_{k}^{(l+1)}=\sigma_{k}^{(l)}+\alpha^{(l)} \sigma_{k}^{(0)} p_{k}^{(l)},
$$

where the step length $\alpha^{(l)}$ is computed by an inexact line search in the model space $\mathbf{m}$. What is crucial in this approach is that it requires (1) relatively small storage proportional to $n_{c p} \times N$, where $n_{c p}$ is the number of the correction pairs, and (2) only the (multiple) calculation of the derivatives rather than the time-consuming sensitivities and/or the Hessian matrices. The essential difficulty of the 3D solution is the calculation of the derivatives:

$$
\frac{\partial \varphi_{d}}{\partial m_{k}}=\frac{\partial \varphi_{d}}{\partial \sigma_{k}} \sigma_{k}^{(0)}
$$

\section{CALCULATION OF DERIVATIVES}

To derive the derivatives $\partial \varphi_{d} / \partial \sigma_{k}(k=1, \ldots, N)$, we apply a technique based on the EM adjoint method (cf. Rodi, 1976). This method uses the EM field reciprocity and has been applied to calculate sensitivities (Weidelt, 1975; McGillivray and Oldenburg, 1990) and for forward modeling and inversion (Dorn et al., 1999; Newman and Alumbaugh, 2000; Rodi and Mackie, 2001; Newman and Boggs, 2004; Chen et al., 2005).

From equation 2, it follows that

$$
\frac{\partial \varphi_{d}}{\partial \sigma_{k}}=\operatorname{Re}\left\{\sum_{j=1}^{N_{S}} \sum_{n=1}^{N_{T}} \beta_{j n} \operatorname{tr}\left[\overline{\mathbf{A}}_{j n}^{T} \frac{\partial \mathbf{Z}_{j n}}{\partial \sigma_{k}}\right]\right\},
$$

where $\mathrm{Re}$ is the real part of the argument. To derive equation 8 from equation 2, one might want to use obvious properties, such as $\partial \mathbf{D}_{j n} / \partial \sigma_{k}=0$, or $\operatorname{tr}[\mathbf{B}]=\operatorname{tr}\left[\mathbf{B}^{T}\right]$ and $\operatorname{tr}[\mathbf{C}+\mathbf{B}]=\operatorname{tr}[\mathbf{C}]+\operatorname{tr}[\mathbf{B}]$ for $\mathbf{C}$ and $\mathbf{B}$. Substituting equation B-5 for equation 8 , one obtains

$$
\frac{\partial \varphi_{d}}{\partial \sigma_{k}}=\operatorname{Re}\left\{\sum_{j=1}^{N_{S}} \sum_{n=1}^{N_{T}} \beta_{j n} \operatorname{tr}\left[\overline{\mathbf{A}}_{j n}^{T}\left(\mathbf{E}_{j n, k}-\mathbf{Z}_{j n} \mathbf{H}_{j n, k}\right) \mathbf{H}_{j n}^{-1}\right]\right\},
$$

where we denote

$$
\mathbf{Z}_{j n, k}=\frac{\partial \mathbf{Z}_{j n}}{\partial \sigma_{k}}, \quad \mathbf{E}_{j n, k}=\frac{\partial \mathbf{E}_{j n}}{\partial \sigma_{k}}, \quad \mathbf{H}_{j n, k}=\frac{\partial \mathbf{H}_{j n}}{\partial \sigma_{k}} .
$$

In Appendix B, we prove that calculating the matrices in equation 10 for the whole set of triple indices $\left\{(j, n, k): j=1, \ldots, N_{S} ; n\right.$ $\left.=1, \ldots, N_{T} ; k=1, \ldots, N\right\}$ requires solving $2 \times N_{T} \times(N+1)$ forward problems (equations B-3 and B-7). Obviously, for a 3D conductivity model where the number of cells $N$ is relatively large, such an approach is impractical. Fortunately, we need to calculate the derivatives $\partial \varphi_{d} / \partial \sigma_{k}(k=1, \ldots, N)$ rather than the matrices of equation 10. As we demonstrate below, significantly fewer forward problems must be solved when calculating derivatives.

Along with the forward problems given in equation B-7, let us consider $2 \times N_{T}$ adjoint problems, presented by Maxwell equations

$$
\nabla \times \mathbf{v}_{n}=\sigma \mathbf{u}_{n}+\mathbf{j}_{n}^{\text {ext }}+\nabla \times \mathbf{h}_{n}^{\text {ext }}
$$

and

$$
\nabla \times \mathbf{u}_{n}=i \omega_{n} \mu \mathbf{v}_{n},
$$

where

$$
\mathbf{j}_{n}^{\text {ext }}=\sum_{j=1}^{N_{S}} \beta_{j n} \mathbf{p}^{T} \overline{\mathbf{A}}_{j n} \mathbf{H}_{j n}^{-T} \delta\left(\mathbf{r}-\mathbf{r}_{j}\right)
$$

and

$$
\mathbf{h}_{n}^{\text {ext }}=-\frac{1}{i \omega_{n} \mu} \sum_{j=1}^{N_{S}} \beta_{j n} \mathbf{p}^{T} \mathbf{Z}_{j n}^{T} \overline{\mathbf{A}}_{j n} \mathbf{H}_{j n}^{-T} \delta\left(\mathbf{r}-\mathbf{r}_{j}\right)
$$

and where $\mathbf{H}_{j n}^{-T}$ means the transpose of $\mathbf{H}_{j n}^{-1}$ and $\delta$ is the Dirac's delta function. In addition,

$$
\mathbf{p}=\left(\begin{array}{lll}
1 & 0 & 0 \\
0 & 1 & 0
\end{array}\right)
$$

is the projection matrix, $n=1, \ldots, N_{T}$, and $i=\sqrt{-1}$. It is proven in Appendix C that

$$
\frac{\partial \varphi_{d}}{\partial \sigma_{k}}=\operatorname{Re}\left\{\sum_{n=1}^{N_{T}} \int_{V_{k}} \operatorname{tr}\left[\mathbf{u}_{n}^{T} \mathbf{E}_{n}\right] d V\right\},
$$

where

$$
\begin{aligned}
\operatorname{tr}\left[\mathbf{u}_{n}^{T} \mathbf{E}_{n}\right]= & u_{x}^{(1)} E_{x}^{(1)}+u_{y}^{(1)} E_{y}^{(1)}+u_{z}^{(1)} E_{z}^{(1)}+u_{x}^{(2)} E_{x}^{(2)} \\
& +u_{y}^{(2)} E_{y}^{(2)}+u_{z}^{(2)} E_{z}^{(2)} .
\end{aligned}
$$

The superscripts 1 and 2 denote the polarization of the source. Equation 14 means, practically, that computational loads for calculating the derivatives $\partial \varphi_{d} / \partial \sigma_{k}(k=1, \ldots, N)$ are equivalent to those for solving $2 \times N_{T}$ forward problems using equation B-3 to find $\mathbf{E}_{n}$ and for solving $2 \times N_{T}$ adjoint problems using equation 11 to find $\mathbf{u}_{n}$ for all $n=1, \ldots, N_{T}$. As mentioned, straightforward calculation of the derivatives using equations 9 and B-6-B-8 would require solving 2 $\times N_{T} \times(N+1)$ forward problems

This approach is quite general. It is not limited to magnetotellurics but can be applied to a variety of EM problems (see Avdeev, 2005).

\section{Numerical verification}

To calculate the derivatives of equation 14 , we need to solve the adjoint system of Maxwell's equations 11. To solve this system, we should be able to calculate not the electric field $\mathbf{u}_{n}$ but its averages over numerical cells $V_{k}$ for the media excited by horizontal electric $\mathbf{j}_{n}^{\text {ext }}$ and magnetic $\mathbf{h}_{n}^{\text {ext }}$ dipoles. The $x 3 \mathrm{D}$ forward modeling code of Avdeev et al. $(1997,2002)$ computes exactly these averages. To verify the ability of $x 3 \mathrm{D}$, we checked it against an analytical solution for a uniform space.

For such a space, the $y$-component of the electric field $\mathbf{u}$ excited by a horizontal magnetic dipole of moment $\left(M_{x}, 0,0\right)$ located at the coordinate origin follows Ward and Hohmann (1987):

$$
u_{y}=-i \omega \mu \frac{M_{x} z a(r)}{r^{2}}(1+\kappa r),
$$

where $\kappa^{2}=-i \omega \mu \sigma_{0}, r=\sqrt{x^{2}+y^{2}+z^{2}}, a(r)=(1 / 4 \pi r) e^{-\kappa r}$ and $\sigma_{0}$ is the conductivity of the space. Using the $x 3 \mathrm{D}$ code, we calculate the 10-s electric field $\mathbf{u}$ for a $100-\mathrm{ohm}-\mathrm{m}$ uniform space. The modeling domain comprises $N_{x} \times N_{y} \times N_{z}=32 \times 32 \times 7=7168$ rectangular prisms, with $d_{x}=d_{y}=1 \mathrm{~km}$. The magnetic dipole is situated in the center of the upper face of the central cell $V_{c}$. For each prism, we compute an average of $\mathbf{u}$ and compare it with the analytical solution of equation 16. This comparison for the $y$-component of the av- 
eraged electric field $\left\langle u_{y}\right\rangle_{k}=\left(1 /\left|V_{k}\right|\right) \int_{V_{k}} u_{y} d V$ is presented in Figure $1 \mathrm{a}$.

To calculate analytically the average $\left\langle u_{y}\right\rangle_{c}$ for the most complicated central prism $V_{c}$ where the dipole is seated, we use the fact that this prism is located at the near zone $|\kappa r| \ll 1$. For the near zone from equation 16 , we approximate the average as

$$
\begin{aligned}
\left\langle u_{y}\right\rangle_{c} & =\frac{1}{\left|V_{c}\right|} \int_{V_{c}} u_{y} d V \\
& \approx i \omega \mu \frac{M_{x}}{2 d_{x} d_{y}}\left(\frac{1+\varepsilon-\sqrt{1+\varepsilon^{2}}}{\varepsilon}\right),
\end{aligned}
$$

where $\varepsilon=d_{z 0} / d, d=\sqrt{d_{x} d_{y} / \pi}$, and $d_{z 0}$ is the thickness of the prism. For other cells, we use the following formula:

$$
\left\langle u_{y}\right\rangle_{k}=u_{y}\left(x_{k}^{c}, y_{k}^{c}, z_{k}^{c}\right),
$$

where $\left(x_{k}^{c}, y_{k}^{c}, z_{k}^{c}\right)$ is the center of the $k$ th cell and $u_{y}$ on the right side is given by equation 16 .

In Figure 1, we present, for the sake of resolution, only $7 \times 7$ cells located around the magnetic dipole for the first seven horizontal slices, 0-7 km depth. One can see very good agreement between results produced by $x 3 \mathrm{D}$ and the analytical solution. We compared the $x 3 \mathrm{D}$ solution and the analytical one for all other components of the electric field $\mathbf{u}$ (not presented here) and found very good agreement.
We performed similar comparisons for the horizontal electric dipole with moment $\left(M_{x}, 0,0\right)$. For this experiment, we used the same uniform space with a resistivity of $100 \mathrm{ohm}-\mathrm{m}$, the same period of $10 \mathrm{~s}$ and the same numerical grid of $N_{x} \times N_{y} \times N_{z}=32 \times 32 \times 7$ $=7168$ rectangular prisms, with $d_{x}=d_{y}=1 \mathrm{~km}$. The analytical expression for the $x$-component of the electric field in this case follows Ward and Hohmann (1987), where

$$
u_{x}=\frac{M_{x} a(r)}{\sigma_{0} r^{2}}\left(\left(3+3 \kappa r+\kappa^{2} r^{2}\right) \frac{x^{2}}{r^{2}}-\left(1+\kappa r+\kappa^{2} r^{2}\right)\right) \text {. }
$$

As mentioned, we need the average values of electric field $\mathbf{u}$ over the prisms. To approximate this average over the central cell (the cell where the electric dipole is situated), we use the following formula:

$$
\left\langle u_{x}\right\rangle_{c}=\frac{1}{\left|V_{c}\right|} \int_{V_{c}} u_{x} d V \approx-\frac{M_{x}}{4 \sigma_{0} d_{x} d_{y}} \frac{1}{\sqrt{d_{z 0}^{2}+d^{2}}} .
$$

This formula is obtained by integrating equation 19 over the prism and assuming the near zone $|\kappa r| \ll 1$. For other cells, again we use

$$
\left\langle u_{x}\right\rangle_{k}=u_{x}\left(x_{k}^{c}, y_{k}^{c}, z_{k}^{c}\right),
$$

where $u_{x}$ on the right side is given by equation 19 . The comparison of $\left\langle u_{x}\right\rangle$ obtained from $x 3 \mathrm{D}$ and from the analytical approximations (equations 20 and 21) is presented in Figure 1b. We performed the comparison for all other components of the electric field and obtained good agreement.

Our conclusion from these experiments is that we can use the $x 3 \mathrm{D}$ code to calculate the derivatives given in equation 14

\section{VALIDATION OF METHOD}

To investigate the robustness and effectiveness of the MT inversion method, we performed several numerical experiments. For all of these experiments, the $x 3 \mathrm{D}$ forward-modeling code was exploited as an inversion engine to solve the forward and adjoint problems given in equations B-3 and 11. It also was used to calculate $2 \times 2$ matrices $\mathbf{D}_{j n}$ of observed impedances. In addition, we added $1 \%$ random noise to these data and assigned the relative errors $\varepsilon_{j n}$ of the impedance, needed to define weights $\beta_{\text {jn }}$ (see equation 2 ), as 0.05 . This value of $\varepsilon_{j n}$ means that the misfit $\varphi_{d}$ defined in equation 2 drops to 1 , when

$$
\begin{aligned}
\mathrm{rms}= & \left(\frac{1}{N_{S} N_{T}} \sum_{j=1}^{N_{S}} \sum_{n=1}^{N_{T}}\right. \\
& \left.\times \frac{\operatorname{tr}\left[\overline{\left.(\mathbf{Z}-\mathbf{D})_{j n}^{T}(\mathbf{Z}-\mathbf{D})_{j n}\right]}\right.}{\operatorname{tr}\left[\overline{\mathbf{D}}_{j n}^{T} \mathbf{D}_{j n}\right]}\right)^{1 / 2}
\end{aligned}
$$

drops to $5 \%$. Using the same forward code for the predicted values and to generate the observed data is sufficient for testing the inversion because the $x 3 \mathrm{D}$ code has been tested against many other 
forward-modeling codes (see Miensopust, 2008) and the difference between the responses is less than the noise we added to the data.

Also, we constrained the conductivity values $\sigma_{k}(k=1, \ldots, N)$ to lie between $l_{k}=10,000 \mathrm{ohm}-\mathrm{m}$ and $u_{k}=0.01 \mathrm{ohm}-\mathrm{m}$ in equation 4 . The number of correction pairs $n_{c p}$ was chosen as six after a series of inversion runs with various values of $n_{c p}$.

\section{Outcropping conductive dike}

Our first model consists of a tilted 3-ohm-m dike embedded into a $100-$ ohm-m half-space. The dike is located at a depth of $0-500 \mathrm{~m}$ and consists of five shifted adjacent blocks of $200 \times 800 \times 100 \mathrm{~m}^{3}$ each. Horizontal $(x-y)$ slices through the model starting from the top (left) to the bottom (right) and vertical $(x-z)$ slice through the center of the model are presented in Figure 2a and b, respectively. A similar model is used to test a 3D MT inversion algorithm in Zhdanov and Tolstaya (2004).

The modeling domain comprises $N_{x} \times N_{y} \times N_{z}=35 \times 35 \times 7$ rectangular prisms (cells) of $100 \times 100 \times 100 \mathrm{~m}^{3}$ in size that cover the dike and some part of the surroundings; it extends from 0 to 700 $\mathrm{m}$ depth. The inversion domain is smaller than the modeling domain and comprises $N_{x} \times N_{y} \times N_{z}=16 \times 24 \times 7$ cells of the same size. This means that $N=2688$ conductivities $\sigma_{k}(k=1, \ldots, N)$ of the prisms need to be recovered. This model is challenging because of numerical difficulties that arise from the outcropping of the dike, and we expect erratic behavior of the recovered conductivities $\sigma_{k}$ in the near-surface layers. Indeed, because it follows from equation 14 to calculate the derivatives $\partial \varphi_{d} / \partial \sigma_{k}(k=1, \ldots, N)$, we have to find the adjoint fields $\mathbf{u}_{n}\left(n=1, \ldots, N_{T}\right)$ and average them over every cell $V_{k}$ of the inversion domain. Equation 11 shows that these adjoint fields $\mathbf{u}_{n}$ are the electric fields generated by electric and magnetic horizontal dipoles. The dipoles are positioned in the locations of the MT sites. For the outcropped dike, some surface cells of the inversion domain touch the dipoles, making the averaging over these cells difficult. A closer examination of the problem shows that it is rooted in the physics of the 3D MT problem: The derivatives of the data misfit for the surface cells touching the dipoles are significantly greater than for all other cells. This reflects the fact that these cells are far more sensitive to the MT data.

Returning to the model, we calculated the observed data, matrices $\mathbf{D}_{j n}$, for $N_{T}=4$ frequencies $\left(f_{n}=1 / T_{n}\right)$ of $1000,100,10$, and $1 \mathrm{~Hz}$ $\left(n=1, \ldots, N_{T}\right)$ and at $N_{S}=42$ sites $r_{j}\left(j=1, \ldots, N_{S}\right)$ coinciding with the nodes of a homogeneous $n_{x} \times n_{y}=6 \times 7$ grid with $200 \mathrm{~m}$ between adjacent nodes (see Figure 3 ). Usually, more frequencies are used for real MT surveys; however, our experiments are mainly for understanding and improving the inversion solution. Using the realistically higher number of frequencies would lead to very long inversion times and therefore fewer experiments.

We start the inversion with the initial-guess model, which has $50 \mathrm{ohm}-\mathrm{m}$ everywhere inside the inversion domain. The result of the inversion is presented in Figure 2. Comparison with the true model shown in the same figure demonstrates that the position, shape, and amplitude of the true anomaly are recovered successfully, although a few resistive artifacts remain. This is especially true for the upper part of the model. As we can expect for an MT inversion, the deeper layers are not recovered as sharply as the upper layers: The bottom part of the recovered model is smeared out naturally, delivering only a hint of the presence of the conductive dike.
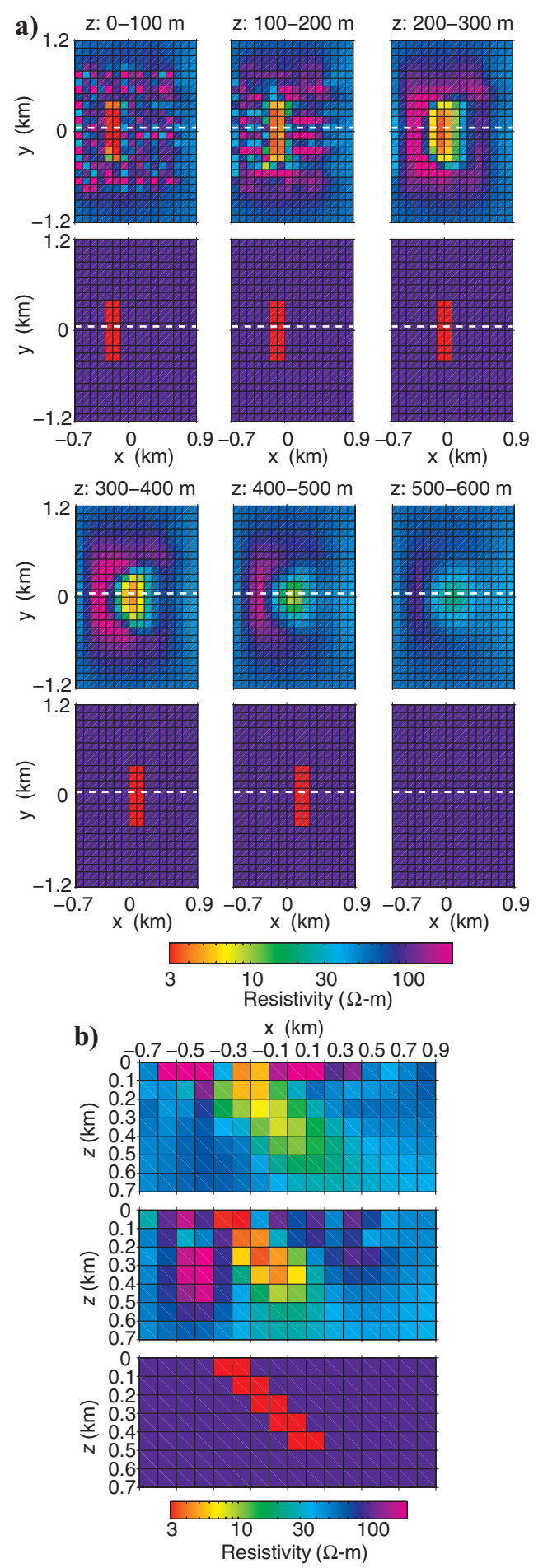

Figure 2. Inversion result for $42 \mathrm{MT}$ sites. (a) Each row of panels presents three horizontal $(x-y)$ slices through the model starting from the top left to the bottom right. The depths of the slices are written above each panel. The first and third rows show an image recovered with the use of four frequencies. The second and fourth rows present the true model. (b) Comparison for a vertical $(x-z)$ cross section. The location of the cross section is shown as a dotted white line in (a). The uppermost panel presents the inversion result obtained with the use of a single frequency; the middle panel shows the image recovered when four frequencies were used; the lower panel presents the true model. 
The convergence curve for this inversion is presented in Figure 4. The curve is shown as a function of the index $n_{f g}$, which increases by one after each evaluation of a pair $\varphi$ and $\nabla_{\mathrm{m}} \varphi$. This index is proportional to the time of the inversion and a little larger than the number of QN iterations. The inversion was terminated when the data misfit $\varphi_{d}$ could not be improved significantly; it dropped to 0.18 at $n_{f g}$ $=550$. As for the regularization parameter, we started with $\lambda=10^{10}$ and then diminished it gradually to $10^{6}$ (see the dashed line). It takes 10 minutes for a single penalty function and its gradient evaluation to be computed on a P4 2.8-GHz/512-RAM laptop. This means it takes 4 days to obtain the result.

We also inverted only $10-\mathrm{Hz}$ responses with $1 \%$ added noise. For this single-frequency experiment, we obtained a blurry image of the conductor at the lower part of the model with a lot of artificial resistive artifacts, especially in the first layer (see Figure 2b, top panel). Although the shape of the dike is recovered in Figure 2, the upper part of it is shifted to the right by one cell. Comparing this recovered image with that obtained with four frequencies (middle panel, Figure $2 \mathrm{~b}$ ), we conclude that an increased number of frequencies, involved in inversion, helps improve the inversion result.

So far we have dealt with a relatively simple problem. Although the results are promising, they give only a first indication of the reliability and stability of the method. Hence, more complicated situations are studied below.

\section{Two adjacent blocks}

The next model has been considered in various 3D forward-modeling papers (e.g., Wannamaker, 1991; Mackie et al., 1994; Avdeev et al., 1997). Moreover, the inversion code by Siripunvaraporn et al. (2005) is tested using this model. The model consists of resistive and conductive adjacent blocks buried in a two-layered earth. The hori-

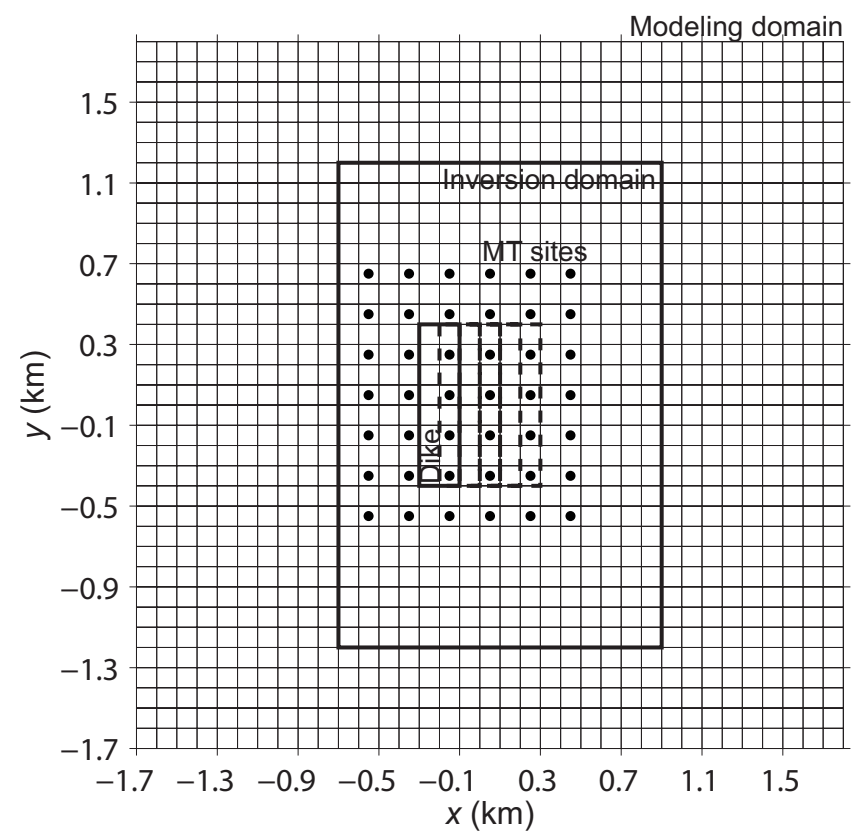

Figure 3. Location of 42 MT sites plotted on top of the numerical grid. The dashed boxes mark the position of the underground conductive blocks of the dike. zontal and vertical slices presented in Figure 5 completely describe the model. The inversion domain consists of $N_{x} \times N_{y} \times N_{z}=20$ $\times 20 \times 9=3600$ rectangular cells with $d_{x}=d_{y}=4000 \mathrm{~m}$ and reaches a depth of $32 \mathrm{~km}$. The modeling domain coincides with the inversion domain.

\section{MT sites}

For our first experiment with this model, we cover the surface $(z$ $=0)$ of the inversion domain with $400 \mathrm{MT}$ sites $\left(N_{S}=400\right)$, located on top of every surface cell of the grid. For these MT sites, we simulate the observed data, matrices $\mathbf{D}_{j n}$, at $N_{T}=3$ frequencies of $10^{-3}$, $3.3 \times 10^{-3}$, and $10^{-2} \mathrm{~Hz}$ and add $1 \%$ noise to the simulated data. Siripunvaraporn et al. (2005) use higher frequencies of $10^{-3}$, $10^{-2}, 10^{-1}, 1$, and $10 \mathrm{~Hz}$. We also use the stabilizer $\varphi_{s}$ and the technique of gradually diminishing regularization parameter $\lambda$ in the inversion. We stop the inversion process when the value of the data misfit $\varphi_{d}$ cannot be improved any more and it drops to 9.7. A single calculation of the penalty function together with its gradient for this experiment takes about 7 minutes on a serial PC, resulting in a total time of 50 hours.

The result of the inversion is shown in Figure 5 along with the true model. The initial-guess model has $50 \mathrm{ohm}-\mathrm{m}$ conductivity in all cells of the inversion domain, assuming that outside conductivity coincides with the true background. For this model, the true background is a two-layer structure with a $10-\mathrm{km}$-thick, $10 \mathrm{ohm}-\mathrm{m}$ layer atop the $100 \mathrm{ohm}-\mathrm{m}$ half-space. Comparing the recovered image with the true model, we obtain a satisfactory result - the shape and position of the blocks are recovered. The value of the resistivity for the conductive block is retrieved correctly, although it is overestimated for the resistive block. As usual for MT inversion, the position of the bottom of the conductive block is somewhat obscured.

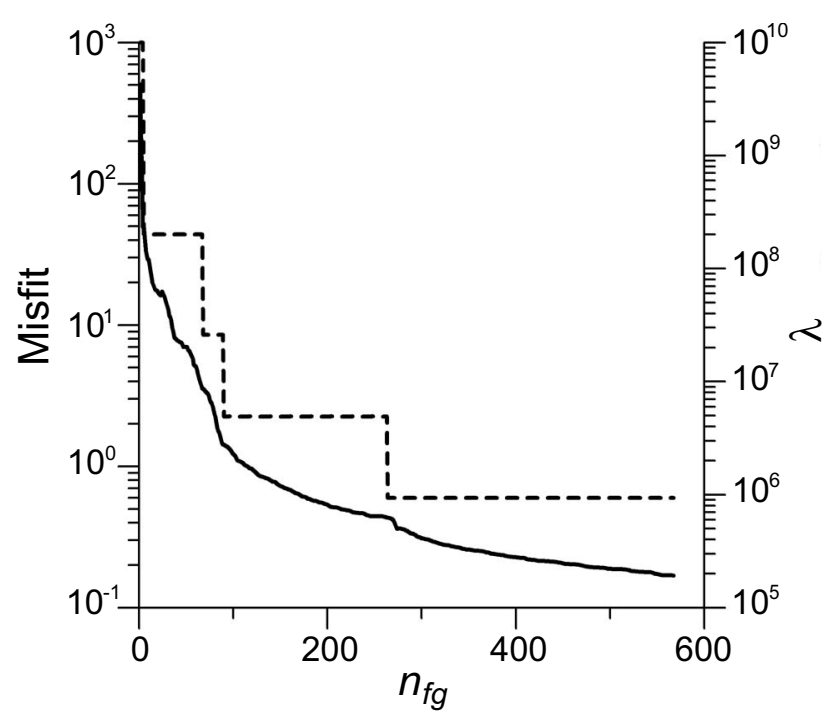

Figure 4. Convergence of the inversion; 42 MT sites and four frequencies were used. The inversion terminates when $\varphi_{d}$ drops to 0.18 , which corresponds to an rms of $2 \%$. Regularization parameter $\lambda$ used for this inversion is shown by the dashed curve. 


\section{MT sites}

Now, we diminish the number of MT sites used for the inversion to 80 . These sites are placed randomly; however, we prevent two sites from being placed in directly adjacent cells. The locations of these MT sites are shown in Figure 6. Everything else is kept as in the previous experiment; we change only the number of MT sites.

Figure 7 presents the result of the inversion. The recovered image is very different from the true model. It has very erratic behavior, especially for the upper part of the model, with many artificial structures. This result cannot be considered satisfactory. If we plot the locations of the MT sites on top of the recovered image (Figure 8), we see that reasonable resistivity values occur exactly for the cells below the MT sites. For these selected cells, it is obviously possible to retrieve the underground structure; at the same time, it is absolutely impossible to use the resistivity of the other cells.

\section{DISCUSSION}

Let us first explain why the 3D MT QN inversion with the constraints imposed by traditional Tikhonov regularization sometimes cannot resolve the resistivity structure immediately beneath the surface in regions not covered by MT sites. To explain this phenomenon, we rewrite equation 7 for the first model update $\boldsymbol{\sigma}^{(1)}$ $=\left(\sigma_{1}^{(1)}, \ldots, \sigma_{N}^{(1)}\right)^{T}$ as

$$
\sigma_{k}^{(1)}=\sigma_{k}^{(0)}\left(1+\alpha^{(0)} p_{k}^{(0)}\right)
$$

Here, $\sigma_{k}^{(0)}$ is the conductivity of the $k$ th cell of the initial guess model. Further,

$$
p_{k}^{(0)}=-\left.\sigma_{k}^{(0)}\left(\frac{\partial \varphi_{d}}{\partial \sigma_{k}}+\lambda \frac{\partial \varphi_{s}}{\partial \sigma_{k}}\right)\right|_{\boldsymbol{\sigma}=\boldsymbol{\sigma}^{(0)}},
$$

as follows from equations 1 and 5 and from the fact that $\mathbf{G}^{(0)}=\mathbf{I}$. Substituting equation 23 into equation 22 , we obtain

$$
\sigma_{k}^{(1)}=\sigma_{k}^{(0)}\left(1-\left.\alpha^{(0)} \sigma_{k}^{(0)}\left(\frac{\partial \varphi_{d}}{\partial \sigma_{k}}+\lambda \frac{\partial \varphi_{s}}{\partial \sigma_{k}}\right)\right|_{\sigma=\boldsymbol{\sigma}^{(0)}}\right)
$$

This expression for the first update $\boldsymbol{\sigma}^{(1)}$ means that the smoothness of $\boldsymbol{\sigma}^{(1)}$ is usually related directly to the smoothness of the gradient

$$
\nabla_{\sigma} \varphi_{d}=\left(\frac{\partial \varphi_{d}}{\partial \sigma_{1}}, \ldots, \frac{\partial \varphi_{d}}{\partial \sigma_{N}}\right)^{T}
$$

Indeed, equation 24 usually can be rewritten as

$$
\sigma_{k}^{(1)}=\sigma_{k}^{(0)}-\left.\alpha^{(0)} \sigma_{k}^{(0)^{2}} \frac{\partial \varphi_{d}}{\partial \sigma_{k}}\right|_{\boldsymbol{\sigma}=\boldsymbol{\sigma}^{(0)}}
$$

because in many cases the initial guess model $\boldsymbol{\sigma}^{(0)}$ is chosen as a uniform half-space and, consequently, $\partial \varphi_{s} /\left.\partial \sigma_{k}\right|_{\boldsymbol{\sigma}=\boldsymbol{\sigma}^{(0)}}=0$.
Our experience with the model of two adjacent blocks and with data from 80 MT sites showed that the first update $\boldsymbol{\sigma}^{(1)}$ looks very rough. Moreover, the smoothness of this image cannot be improved
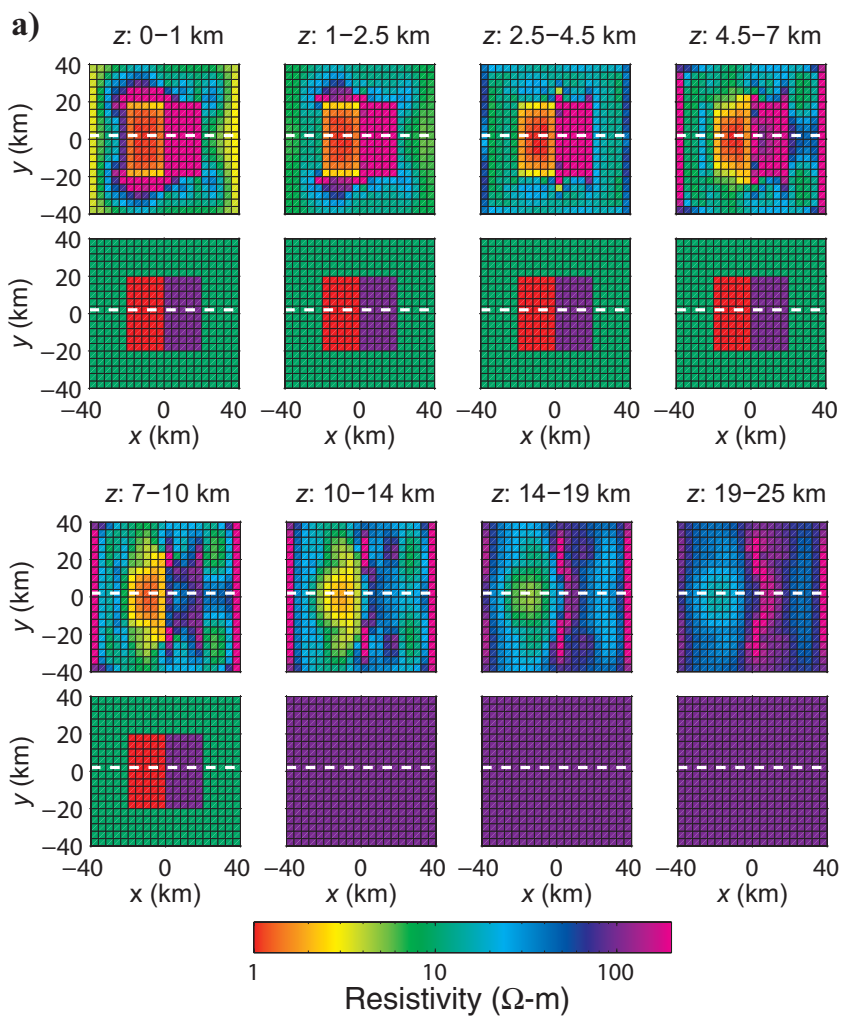

b)
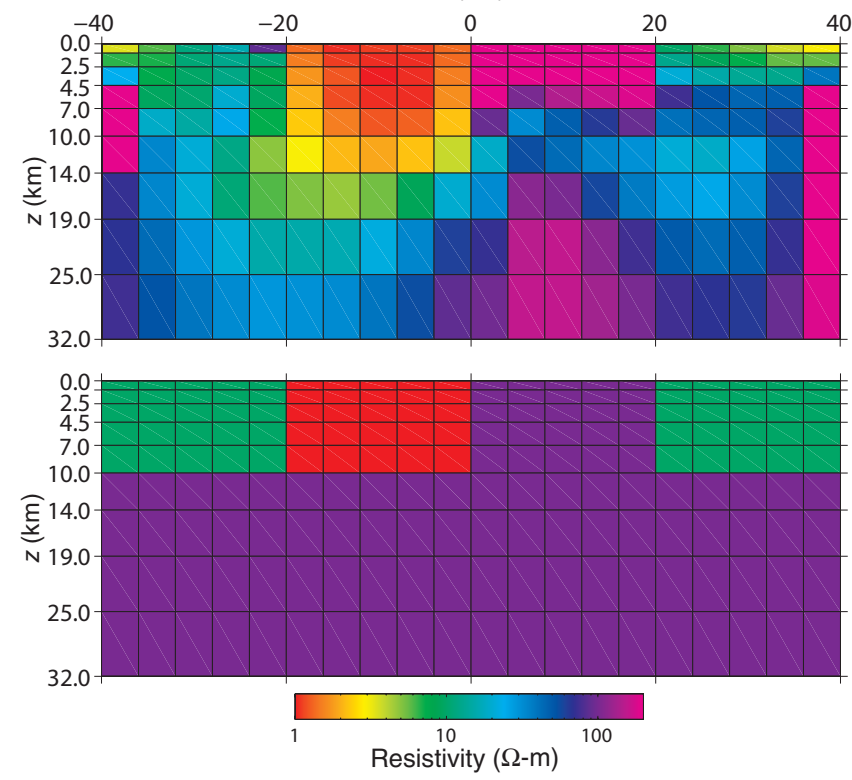

Figure 5. Result of the inversion for 400 MT sites and three frequencies. (a) Each row presents four horizontal $(x-y)$ slices through the model starting from the top left to the bottom right. The first and third rows correspond to the result of the inversion. The second and fourth rows correspond to the true model. (b) Comparison for $(x-z)$ cross section. The location of the cross section is shown as a dotted white line in Figure 5a. The upper panel presents the inversion result; the lower panel presents true model. 
by Tikhonov regularization. This conclusion follows from equation 25 because the right-hand side of the equation does not depend on $\varphi_{s}$. Regularization might help improve this erratic image of $\boldsymbol{\sigma}^{(1)}$ in the course of consequent iterations $\boldsymbol{\sigma}^{(l)}$. In our experience, this type of regularization does not always help, and its effectiveness depends on many factors.

\section{Additional regularization}

The singularity of the gradient $\nabla_{\sigma} \varphi_{d}$ in the vicinity of the MT sites could introduce erratic structures to the model; this behavior complicates the solution of the 3D MT inverse problem. This is particularly true with Newton optimization approaches that rely heavily on gradients. Although the problem outlined above reflects the physics of the 3D MT inverse problem, it is not very well reported in literature - merely hinted at. A general solution is to put more constraints on the model conductivity values directly rather than impose them through the Tikhonov stabilizer $\varphi_{s}$.

As an example of such an approach, Siripunvaraporn et al. (2005) propose to put additional constraints on the resistivity of the cells using the so-called model covariance matrix. Plessix and Mulder (2008) suggest using exponential depth weighting. Alternatively, Mackie et al. (2001, 2007) and Newman and Boggs (2004) propose to adjust the gradient using a Hessian matrix. We propose a simpler approach that could help eliminate the erratic behavior at the upper part of the model.

In the model space $\mathbf{m}=\left(m_{1}, \ldots, m_{N}\right)^{T}$, we introduce a vector $\widetilde{\mathbf{g}}^{(l)}$ $=\left(\widetilde{g}_{1}^{(l)}, \ldots, \widetilde{g}_{N}^{(l)}\right)^{T}$ as

$$
\widetilde{g}_{k}^{(l)}=\sum_{k^{\prime}=1}^{N} f_{k^{\prime} k} \frac{\partial \varphi^{(l)}}{\partial m_{k^{\prime}}},
$$

Figure 6. Location of 80 randomly distributed MT sites plotted on top of a numerical grid. where the coefficients $f_{k^{\prime} k}$ form a positive definite symmetric matrix and $m_{k}=\sigma_{k} / \sigma_{k}^{(0)}$. Furthermore, we modify the QN sequence given in equation 7 , so

$$
\tilde{m}_{k}^{(l+1)}=\tilde{m}_{k}^{(l)}+\alpha^{(l)} \tilde{p}_{k}^{(l)},
$$

where new search direction $\widetilde{\mathbf{p}}^{(l)}=-\widetilde{\mathbf{G}}^{(l)} \widetilde{\mathbf{g}}^{(l)}$ and $\widetilde{m}_{k}^{(0)}=m_{k}^{(0)}$. The matrix $\widetilde{\mathbf{G}}^{(l)}$ is updated at every iteration using the limited-memory BFGS formula, where gradients $\nabla_{\mathbf{m}} \varphi$ should be substituted by $\widetilde{\mathbf{g}}$ and $\widetilde{\mathbf{G}}^{(0)}=\mathbf{I}$.

We choose $f_{k^{\prime} k}$ to be as follows:

$$
f_{k^{\prime} k}=\left\{\begin{array}{c}
\frac{e^{-\frac{1}{2}\left[\left(\frac{i_{x}^{\prime}-i_{x}}{a_{x}}\right)^{2}+\left(\frac{i_{y}^{\prime}-i_{y}}{a_{y}}\right)^{2}\right]}}{\sum_{l_{x}=1}^{N_{x}} \sum_{l_{y}=1}^{N_{y}} e^{-\frac{1}{2}\left[\left(\frac{l_{x}-i_{x}}{a_{x}}\right)^{2}+\left(\frac{l_{y}-i_{y}}{a_{y}}\right)^{2}\right]}, \quad i_{z}=i_{z}^{\prime}} \\
0, \quad i_{z} \neq i_{z}^{\prime}
\end{array}\right.
$$

where $k=\gamma\left(i_{x}, i_{y}, i_{z}\right), k^{\prime}=\gamma\left(i_{x}^{\prime}, i_{y}^{\prime}, i_{z}^{\prime}\right)$, and $\gamma\left(i_{x}, i_{y}, i_{z}\right)=i_{z}+\left[i_{y}\right.$ $\left.-1+\left(i_{x}-1\right) \times N_{y}-1\right] \times N_{z}$. The transformation given in equation 26 is called additional regularization.

\section{Model check}

Now, we can check on how the additional regularization given in equation 26 helps in the example of two adjacent blocks, which was introduced above. As before, the inversion domain coincides with the modeling domain and comprises $N_{x} \times N_{y} \times N_{z}=20 \times 20 \times 9$ $=3600$ rectangular cells, extending to a depth of $32 \mathrm{~km}$. Again, we cover the surface $(z=0)$ of the inversion domain with 80 MT sites $\left(N_{S}=80\right)$. The coordinates of these sites are exactly the same as before (see Figure 6). For these sites, we simulate data at frequencies of $10^{-3}, 3.3 \times 10^{-3}$, and $10^{-2} \mathrm{~Hz}\left(N_{T}=3\right)$ and add $1 \%$ noise to the simulated data.

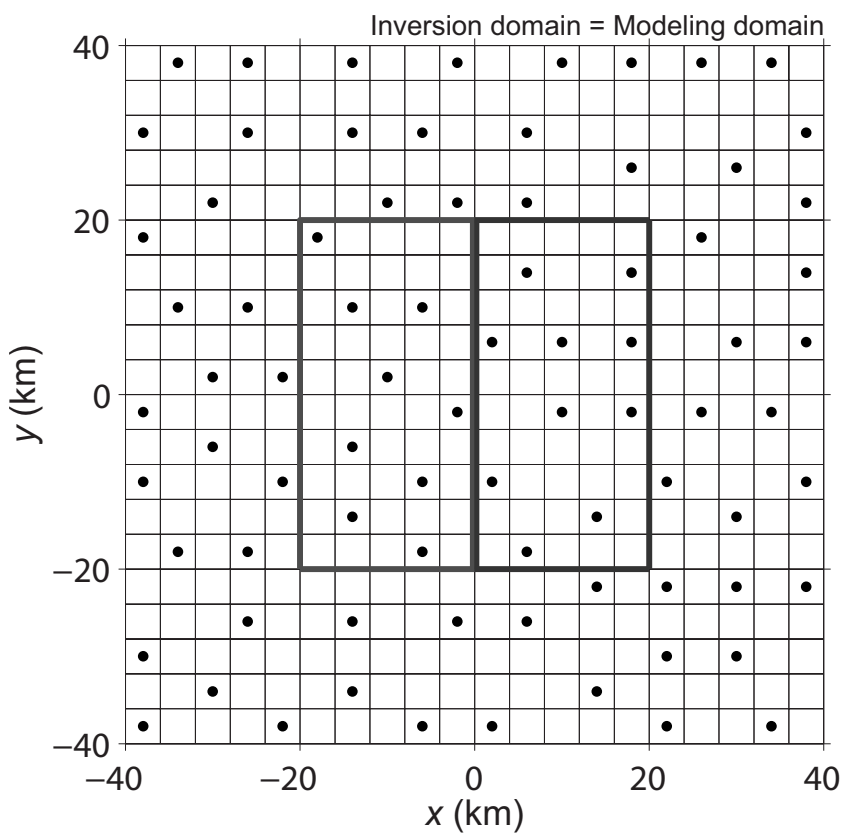


For these data, we compare results from two versions of our inverse problem solution, one without the additional regularization $\left(\nabla_{\sigma} \varphi^{(l)}\right.$ used as the gradient) and another with the additional regularization $\left(\widetilde{\mathbf{g}}^{(l)}\right.$ used as the gradient). Our initial-guess conductivity is $50 \mathrm{ohm}-\mathrm{m}$ for the whole inversion domain, assuming that the outside conductivity coincides with the true background for both of these solutions.

For the inversion with the additional regularization, we introduce two extra parameters $a_{x}$ and $a_{y}$ in the right-hand side of equation 28. Values of these parameters that are too large deliver an overly smooth resistivity image, not allowing a sufficiently small data misfit $\varphi_{d}$. Therefore, to reach a satisfactory value of the data misfit, we use a sequence of decreasing parameters $a_{x}$ and $a_{y}$, similar to the reduction of the regularization parameter $\lambda$.

First, we choose $a_{x}=a_{y}=3$ and then gradually diminish the values of these parameters. With the first values, we run 10 iterations, change them to $a_{x}=a_{y}=2$ for 20 iterations, then $a_{x}=a_{y}=1$ for an additional 20 iterations, and finally the final 100 iterations without regularization. We tried various values of $a_{x}$ and $a_{y}$, but all of these experiments show that the exact values are not critical for the inversion results.

Comparison of the inversion results with and without additional regularization is shown in Figure 7. The result with the additional regularization is much more similar to the true model. Positions and resistivity values of the anomalies are reasonably well matched. The location of the interface between conductor and resistor is found. With depth, the image becomes smoother and the conductor extends slightly deeper, but this can be expected for MT.

In Figure 9, we compare the convergence curves for the inversions with and without the additional regularization. The inversion without the additional regularization converges to a data misfit $\varphi_{d}$ of 11. The data misfit for the inversion with the additional regularization drops to 2.5 , resulting in a total time of 18.5 hours on a P4 2.8-GHz/512-RAM laptop. A single calculation of the penalty function together with its gradient takes about 7 minutes.
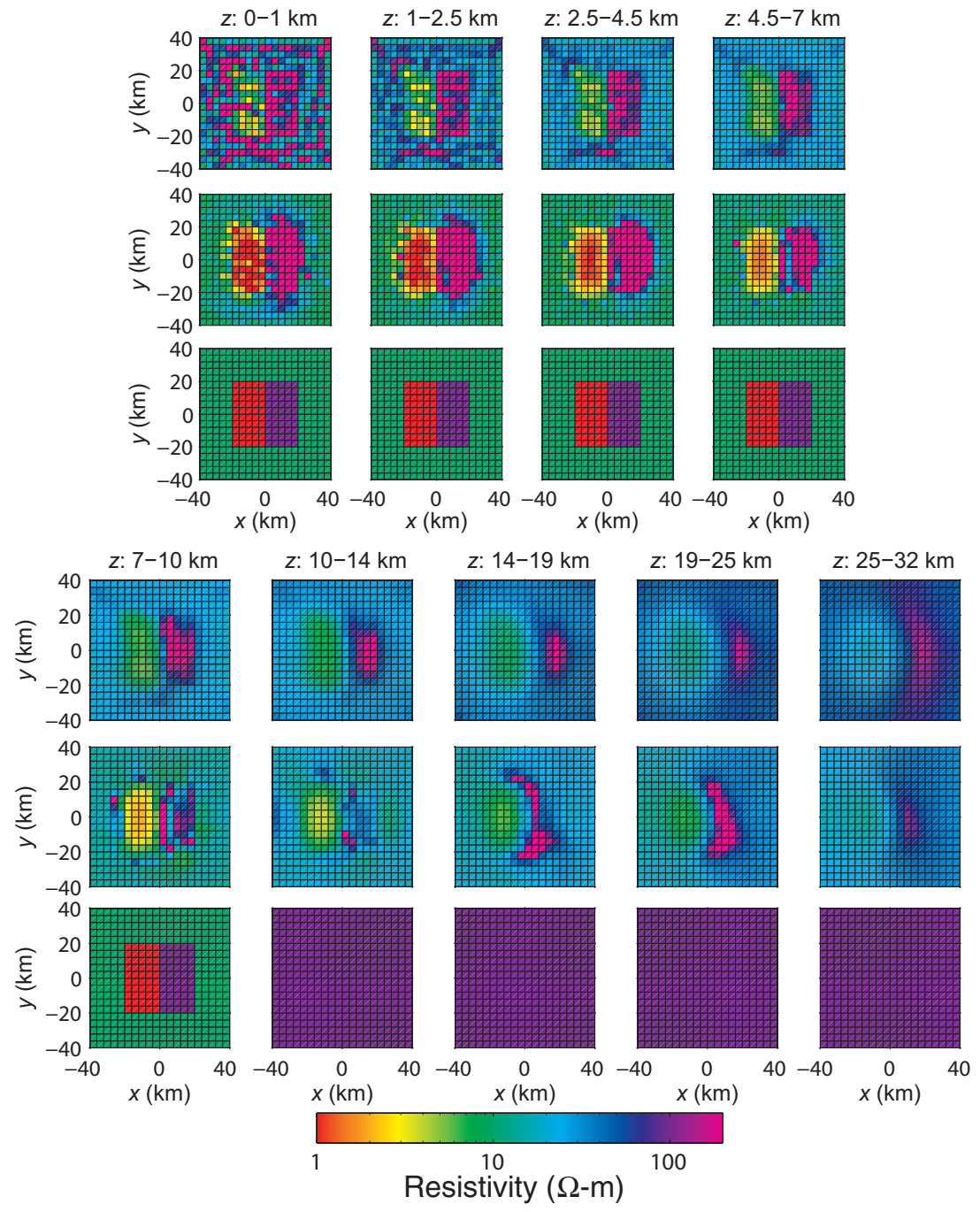

Figure 7. Comparison of the inversion results with and without additional regularization. Each row presents horizontal $(x-y)$ slices through the model starting from the top left to the bottom right. The first and fourth rows correspond to the result of the inversion without additional regularization; the second and fifth rows correspond to the inversion with the additional regularization; and the third and sixth rows are the true model. Three frequencies and $80 \mathrm{MT}$ sites were used. 


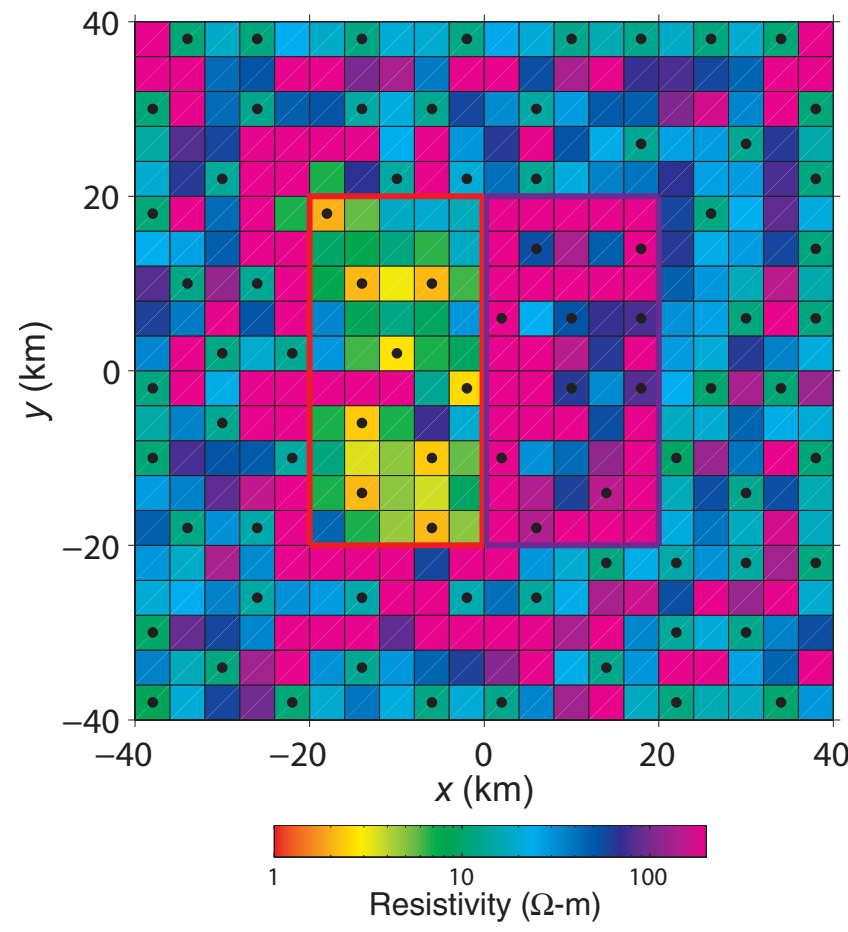

Figure 8. The location of $80 \mathrm{MT}$ sites plotted on top of the upper layer of the recovered image.

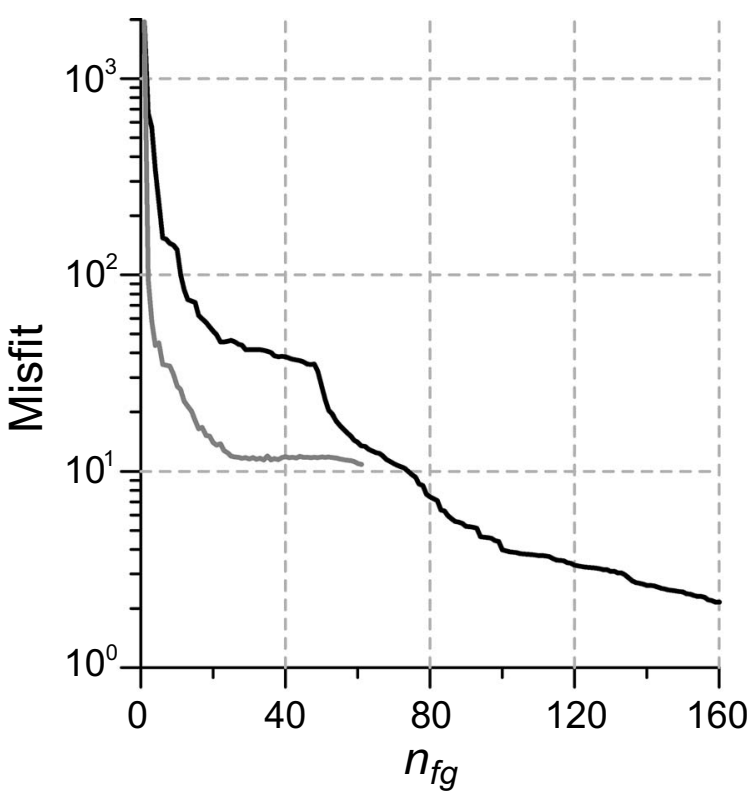

Figure 9. Convergence curves for the inversions without (gray line) and with (black line) additional regularization described in equations 26 and 28. Three frequencies and 80 MT sites were used.

\section{CONCLUSION}

We have developed a novel approach to 3D MT inversion. Our approach is based on a limited-memory QN optimization method. The main advantage of this method, compared to other Newton optimization techniques, is the storage requirement: Only $n_{c p}$ pairs of vec- tors must be stored in memory. This advantage makes it possible to handle large-scale problems, such as 3D MT inversion. As for most other types of optimization methods, the limited-memory QN optimization requires calculation of the gradient of the penalty function $\varphi$.

We developed and implemented the adjoint method to derive explicit expressions for calculating the gradients of the data misfit. Our development is quite general and is not limited to magnetotellurics alone. It can be applied to a variety of EM problems, such as marine controlled-source EM and well induction logging. Because the solution of the 3D MT inverse problem is nonunique, we need an appropriate regularization approach. We suggest Tikhonov regularization, which is based on the finite-difference approximation of the Laplace operator, assuming continuity of the gradient at the boundary of the inversion domain. Another important part of our inversion technique is the choice of the regularization parameter $\lambda$.

Our synthetic tests with a suite of standard models demonstrate that with pure Tikhonov regularization we achieve satisfactory results only with dense site coverage. Generally speaking, though, we are satisfied by the software developed and the results of our model experiments. For the conductive dike model, for example, we can recover reasonably the true resistivity image. For the more complicated model with two adjacent blocks, our findings are controversial. On the one hand, we achieve relatively good results with dense MT sites coverage. At the same time, for a coarser coverage our inversion solution cannot "see" through numerical cells not covered by MT sites. This conclusion implies that the Tikhonov regularization, which we include in our inversion solution, is not powerful enough to suppress the nonsmoothness of the resistivity image, especially for the upper part of the model. To construct reliable resistivity images, one must put stronger constraints on the model parameters stronger than those initially imposed by traditional Tikhonov regularization. We suggest using an additional regularization based on smoothing the gradients of the penalty function. Applying such an approach improves the results dramatically, although some artifacts are still present. A possible explanation is that our regularization is too simple and a more complicated one must be applied.

In the future, a closer examination of the implemented regularization and an investigation of possible alternatives could show how much we can improve the efficiency of our approach. One extension would be applying the automatic relaxation scheme to the regularization. So far, we adjust the value of the regularization parameter $\lambda$ manually at different stages of the inversion, based on our experience. This is time consuming because the inversion must be stopped and the convergence examined manually and restarted with the new value. An automatic scheme would accelerate this process, even though human experience and judgment can never be replaced fully.

Another important improvement would be introducing the static shift into the penalty function of equation 1 . We also plan to apply our inversion scheme to an experimental data set. However, previous examples from other 3D MT inversion software developers indicate that successful verification of the inversion technique even on a single practical data set is a complex task and might take some time.

\section{ACKNOWLEDGMENTS}

This work was supported by the Cosmogrid project, funded by the Programme for Research in Third Level Institutions under the National Development Plan and with assistance from the European Regional Development Fund. We are thankful to Gregory Newman, 
Weerachai Siripunvaraporn, Randall Mackie, Colin Farquharson, and Chester Weiss for their detailed comments and suggestions that improved the manuscript. Thanks to Brian O'Reilly for English corrections. A. Avdeeva acknowledges the constructive support of her supervisors, Alan Jones and Colin Brown.

\section{APPENDIX A}

\section{HOW TO MEASURE THE MISFIT}

The predicted $\mathbf{Z}$ and observed $\mathbf{D}$ impedances (at a given MT site and at a discrete period) are $2 \times 2$ matrices, not scalars. So the question is how to measure the distance $\rho(\mathbf{Z}, \mathbf{D})$ between them to define a proper form of misfit $\varphi_{d}$. The answer is not obvious. A natural way to define such a distance is to consider the matrix $\mathbf{A}=\mathbf{Z}-\mathbf{D}$ and its induced matrix norm

$$
\|\mathbf{A}\|_{2}=\max \frac{\|\mathbf{A} \mathbf{u}\|_{2}}{\|\mathbf{u}\|_{2}},
$$

where $\|\mathbf{u}\|_{2}=\sqrt{\left|u_{1}\right|^{2}+\left|u_{2}\right|^{2}}$ for any vector $\mathbf{u}=\left(u_{1}, u_{2}\right)^{T}$, and define $\rho(\mathbf{Z}, \mathbf{D})=\|\mathbf{Z}-\mathbf{D}\|_{2}$. It can be shown that for any matrix $\mathbf{A}$,

$$
\|\mathbf{A}\|_{2}=\sqrt{\lambda_{1}},
$$

where $\lambda_{1}$ is the largest (real) eigenvalue of Hermitian matrix $\overline{\mathbf{A}}^{T} \mathbf{A}$. Moreover, for a $2 \times 2$ matrix $\mathbf{A}$, it follows that

$$
\left(\|\mathbf{A}\|_{2}\right)^{2}=\frac{1}{2}\left(\operatorname{tr}\left[\overline{\mathbf{A}}^{T} \mathbf{A}\right]+\sqrt{\operatorname{tr}\left[\overline{\mathbf{A}}^{T} \mathbf{A}\right]^{2}-4 \operatorname{det}\left[\overline{\mathbf{A}}^{T} \mathbf{A}\right]}\right),
$$

where

$$
\operatorname{tr}\left[\overline{\mathbf{A}}^{T} \mathbf{A}\right]=\left|A_{x x}\right|^{2}+\left|A_{x y}\right|^{2}+\left|A_{y x}\right|^{2}+\left|A_{y y}\right|^{2}
$$

and $\operatorname{det}\left[\overline{\mathbf{A}}^{T} \mathbf{A}\right]$ are, respectively, the trace and determinant of $\overline{\mathbf{A}}^{T} \mathbf{A}$

Theoretically, we are looking for equation A-3. But it is too complicated (i.e., not quadratic) to be considered as a proper form of the misfit function. Somehow, we must simplify it.

From equation A-3 it follows that

$$
\frac{1}{2} \operatorname{tr}\left[\overline{\mathbf{A}}^{T} \mathbf{A}\right] \leq\left(\|\mathbf{A}\|_{2}\right)^{2} \leq \operatorname{tr}\left[\overline{\mathbf{A}}^{T} \mathbf{A}\right]
$$

The inequalities given in equation A-5 mean that distance $\rho(\mathbf{Z}, \mathbf{D})$ $=\|\mathbf{Z}-\mathbf{D}\|_{2}$ is controlled by the $\operatorname{trace} \operatorname{tr}\left[\overline{\mathbf{A}}^{T} \mathbf{A}\right]$, where $\mathbf{A}=\mathbf{Z}-\mathbf{D}$. This trace can be chosen to measure the misfit as

$$
\varphi_{d}=\frac{1}{2} \operatorname{tr}\left[\overline{\mathbf{A}}^{T} \mathbf{A}\right]
$$

although $\operatorname{tr}\left[\overline{\mathbf{A}}^{T} \mathbf{A}\right]$ is not associated with any matrix norm itself.

\section{APPENDIX B}

\section{MT IMPEDANCE AND ITS DERIVATIVE}

The MT impedance $\mathbf{Z}_{j n}=\mathbf{Z}\left(\mathbf{r}_{j}, \omega_{n}\right)$ at the $j$ th site $\mathbf{r}_{j}$ and the $n$th frequency $\omega_{n}$ is defined as a $2 \times 2$ matrix

$$
\mathbf{Z}_{j n}=\left(\begin{array}{cc}
Z_{x x} & Z_{x y} \\
Z_{y x} & Z_{y y}
\end{array}\right)_{j n}
$$

that satisfies the matrix equation as

$$
\mathbf{E}_{j n}=\mathbf{Z}_{j n} \mathbf{H}_{j n},
$$

where $\mathbf{r}_{j}=\left(x_{j}, y_{j}, z_{j}\right)$ is the position of $j$ th MT site $\left(j=1, \ldots, N_{S}\right)$ and $\omega_{n}=2 \pi / T_{n}$ is the $n$th frequency $\left(n=1, \ldots, N_{T}\right)$. Matrices $\mathbf{E}_{j n}$ and $\mathbf{H}_{j n}$ of equation B-1 are defined as

$$
\mathbf{E}_{j n}=\mathbf{p E}_{n}\left(\mathbf{r}_{j}\right), \quad \mathbf{H}_{j n}=\mathbf{p} \mathbf{H}_{n}\left(\mathbf{r}_{j}\right),
$$

where

$$
\mathbf{p}=\left(\begin{array}{lll}
1 & 0 & 0 \\
0 & 1 & 0
\end{array}\right)
$$

is the projection matrix and where

$$
\mathbf{E}_{n}(\mathbf{r})=\left(\begin{array}{lll}
E_{x}^{(1)} & E_{y}^{(1)} & E_{z}^{(1)} \\
E_{x}^{(2)} & E_{y}^{(2)} & E_{z}^{(2)}
\end{array}\right)_{n}^{T}
$$

and

$$
\mathbf{H}_{n}(\mathbf{r})=\left(\begin{array}{ccc}
H_{x}^{(1)} & H_{y}^{(1)} & H_{z}^{(1)} \\
H_{x}^{(2)} & H_{y}^{(2)} & H_{z}^{(2)}
\end{array}\right)_{n}^{T}
$$

are functions of Cartesian coordinates $\mathbf{r}=(x, y, z)$. Here, the superscript 1 or 2 denotes polarization of the source

$$
\mathbf{J}_{n}(\mathbf{r})=\left(\begin{array}{lll}
J_{x}^{(1)} & J_{y}^{(1)} & J_{z}^{(1)} \\
J_{x}^{(2)} & J_{y}^{(2)} & J_{z}^{(2)}
\end{array}\right)_{n}^{T},
$$

superscript $T$ means transpose, and vectors $\mathbf{E}=\left(E_{x}, E_{y}, E_{z}\right)^{T}$ and $\mathbf{H}$ $=\left(H_{x}, H_{y}, H_{z}\right)^{T}$ are electric and magnetic fields. By definition, 3 $\times 2$ matrices $\mathbf{E}_{n}(\mathbf{r})$ and $\mathbf{H}_{n}(\mathbf{r})\left(n=1, \ldots, N_{T}\right)$ are composed of EM fields; hence, they satisfy $2 \times N_{T}$ systems of Maxwell's equations, written as

$$
\nabla \times \mathbf{H}_{n}=\sigma(\mathbf{r}) \mathbf{E}_{n}+\mathbf{J}_{n}
$$

and

$$
\nabla \times \mathbf{E}_{n}=i \omega_{n} \mu \mathbf{H}_{n}
$$

where $\nabla \times \mathbf{H}_{n}$ and $\nabla \times \mathbf{E}_{n}$ denote $3 \times 2$ matrices

$$
\left(\begin{array}{c}
\nabla \times \mathbf{H}^{(1)} \\
\nabla \times \mathbf{H}^{(2)}
\end{array}\right)_{n}^{T}, \quad\left(\begin{array}{c}
\nabla \times \mathbf{E}^{(1)} \\
\nabla \times \mathbf{E}^{(2)}
\end{array}\right)_{n}^{T},
$$

respectively, with $n=1, \ldots, N_{T}$. From equation B-1, it immediately follows that

$$
\mathbf{Z}_{j n}=\mathbf{E}_{j n} \mathbf{H}_{j n}^{-1}
$$

where $\mathbf{H}_{j n}^{-1}$ is the inverse of matrix $\mathbf{H}_{j n}$. Applying the chain rule of differentiation to equation $\mathrm{B}-4$, one can derive

$$
\mathbf{Z}_{j n, k}=\left(\mathbf{E}_{j n, k}-\mathbf{Z}_{j n} \mathbf{H}_{j n, k}\right) \mathbf{H}_{j n}^{-1},
$$

where we denote $\mathbf{Z}_{j n, k}=\partial \mathbf{Z}_{j n} / \partial \sigma_{k}, \mathbf{E}_{j n, k}=\partial \mathbf{E}_{j n} / \partial \sigma_{k}$, and $\mathbf{H}_{j n, k}$ $=\partial \mathbf{H}_{j n} / \partial \sigma_{k}(k=1, \ldots, N)$. Further, from equations B-2 and B-3 it follows that 


$$
\mathbf{E}_{j n, k}=\mathbf{p e}_{n k}\left(\mathbf{r}_{j}\right), \quad \mathbf{H}_{j n, k}=\mathbf{p h}_{n k}\left(\mathbf{r}_{j}\right),
$$

where $3 \times 2$ matrices $\mathbf{e}_{n k}(\mathbf{r})$ and $\mathbf{h}_{n k}(\mathbf{r})$ satisfy $2 \times N_{T} \times N$ systems of the Maxwell equations

$$
\nabla \times \mathbf{h}_{n k}=\sigma(\mathbf{r}) \mathbf{e}_{n k}+\mathbf{j}_{n k}
$$

and

$$
\nabla \times \mathbf{e}_{n k}=i \omega_{n} \mu \mathbf{h}_{n k},
$$

with given electric current densities

$$
\mathbf{j}_{n k}=\chi_{k} \mathbf{E}_{n} .
$$

To derive equations B-7 and B-8 from equation B-3, one might wish to decompose conductivity as $\sigma(\mathbf{r})=\sum_{k=1}^{N} \sigma_{k} \chi_{k}(\mathbf{r})$, where

$$
\chi_{k}(\mathbf{r})=\left\{\begin{array}{l}
1, \mathbf{r} \in V_{k} \\
0, \mathbf{r} \notin V_{k}
\end{array}\right.
$$

and $V_{k}$ is the volume occupied by the $k$ th cell and then differentiate equations B-3 over $\sigma_{k}$. We assume that $\partial \mathbf{J}_{n} / \partial \sigma_{k}=0$. In equations B-7 and B-8, matrices

$$
\mathbf{e}_{n k}=\left(\begin{array}{ccc}
e_{x}^{(1)} & e_{y}^{(1)} & e_{z}^{(1)} \\
e_{x}^{(2)} & e_{y}^{(2)} & e_{z}^{(2)}
\end{array}\right)_{n k}^{T}
$$

and

$$
\mathbf{h}_{n k}=\left(\begin{array}{lll}
h_{x}^{(1)} & h_{y}^{(1)} & h_{z}^{(1)} \\
h_{x}^{(2)} & h_{y}^{(2)} & h_{z}^{(2)}
\end{array}\right)_{n k}^{T}
$$

are functions of Cartesian coordinates $\mathbf{r}=(x, y, z)$. Thus, to calculate the MT impedance derivatives $\partial \mathbf{Z} / \partial \sigma_{k}\left(\mathbf{r}_{j}, \omega_{n}\right)$ for the whole set of triple indices $\left\{(j, n, k): j=1, \ldots, N_{S} ; n=1, \ldots, N_{T} ; k\right.$ $=1, \ldots, N\}$ one should solve $2 \times N_{T} \times(N+1)$ forward problems given in equations B-3 and B-7.

\section{APPENDIX C}

\section{ADJOINT METHOD}

In this appendix, we derive the key equation 14 . To do this, we rewrite equations B-7 and 11 as

$$
\nabla \times \nabla \times \mathbf{e}_{n k}-i \omega_{n} \mu \sigma(\mathbf{r}) \mathbf{e}_{n k}=i \omega_{n} \mu \chi_{k} \mathbf{E}_{n} \quad(\mathrm{C}-1)
$$

and

$$
\nabla \times \nabla \times \mathbf{u}_{n}-i \omega_{n} \mu \sigma(\mathbf{r}) \mathbf{u}_{n}=i \omega_{n} \mu\left(\mathbf{j}_{n}^{\mathrm{ext}}+\nabla \times \mathbf{h}_{n}^{\mathrm{ext}}\right) .
$$

Multiplying equation C- 1 by $\mathbf{u}_{n}^{T}$ and equation C-2 by $\mathbf{e}_{n k}^{T}$ and integrating the difference of the resulting equations over the whole $3 \mathrm{D}$ space, we obtain

$$
\int_{R^{3}} \operatorname{tr}\left[\mathbf{e}_{n k}^{T} \mathbf{j}_{n}^{\text {ext }}+\mathbf{e}_{n k}^{T} \nabla \times \mathbf{h}_{n}^{\text {ext }}\right] d V=\int_{V_{k}} \operatorname{tr}\left[\mathbf{u}_{n}^{T} \mathbf{E}_{n}\right] d V,
$$

where $V_{k}$ is the volume occupied by the $k$ th cell, $d V=d x d y d z$. To derive equation $\mathrm{C}-3$, one might wish to use the known Green's formula
$\int_{R^{3}}\left(\mathbf{v}^{T} \nabla \times \mathbf{w}-\mathbf{w}^{T} \nabla \times \mathbf{v}\right) d V=0$, which is valid for any complexvalued vector fields $\mathbf{v}$ and $\mathbf{w}$. Then, we modify the left side of equation C-3 as

$$
\int_{R^{3}} \operatorname{tr}\left[\mathbf{e}_{n k}^{T} \mathbf{j}_{n}^{\text {ext }}+i \omega_{n} \mu \mathbf{h}_{n k}^{T} \mathbf{h}_{n}^{\text {ext }}\right] d V=\int_{V_{k}} \operatorname{tr}\left[\mathbf{u}_{n}^{T} \mathbf{E}_{n}\right] d V,
$$

using $\operatorname{tr}\left[\mathbf{e}_{n k}^{T} \nabla \times \mathbf{h}_{n}^{\text {ext }}\right]=\operatorname{tr}\left[\left(\nabla \times \mathbf{e}_{n k}\right)^{T} \mathbf{h}_{n}^{\text {ext }}\right]=i \omega_{n} \mu \operatorname{tr}\left[\mathbf{h}_{n k}^{T} \mathbf{h}_{n}^{\text {ext }}\right]$. Substituting equations 12 and 13 for sources $\mathbf{j}_{n}^{\text {ext }}$ and $\mathbf{h}_{n}^{\text {ext }}$ into equation C-4 and using the property that $\int_{R^{3}} \mathbf{v}(\mathbf{r}) \delta\left(\mathbf{r}-\mathbf{r}_{j}\right) d V=\mathbf{v}\left(\mathbf{r}_{j}\right)$ for any vector field $\mathbf{v}$, one derives

$$
\begin{gathered}
\sum_{j=1}^{N_{S}} \beta_{j n} \operatorname{tr}\left[\overline{\mathbf{A}}_{j n}^{T}\left(\mathbf{p} \mathbf{e}_{n k}\left(\mathbf{r}_{j}\right)-\mathbf{Z}_{j n} \mathbf{p h}_{n k}\left(\mathbf{r}_{j}\right)\right) \mathbf{H}_{j n}^{-1}\right] \\
\quad=\int_{V_{k}} \operatorname{tr}\left[\mathbf{u}_{n}^{T} \mathbf{E}_{n}\right] d V
\end{gathered}
$$

because $\operatorname{tr}[\mathbf{B C}]=\operatorname{tr}[\mathbf{C B}], \operatorname{tr}\left[\mathbf{B}^{T}\right]=\operatorname{tr}[\mathbf{B}]$, and $(\mathbf{B C})^{T}=\mathbf{C}^{T} \mathbf{B}^{T}$ for any pair of matrices $\mathbf{B}$ and $\mathbf{C}$. Comparing equations $\mathbf{C}-5$, B-6, and 9, one concludes immediately that

$$
\frac{\partial \varphi_{d}}{\partial \sigma_{k}}=\operatorname{Re}\left\{\sum_{n=1}^{N_{T}} \int_{V_{k}} \operatorname{tr}\left[\mathbf{u}_{n}^{T} \mathbf{E}_{n}\right] d V\right\} .
$$

\section{APPENDIX D}

\section{A MORE GENERAL MISFIT AND ITS DERIVATIVE}

Equation 2 is written for a particular problem where the data misfit $\varphi_{d}$ includes the impedance difference matrices $\mathbf{A}_{j n}$, whose entries are weighted equally by the values $\sqrt{\beta_{j n}}$. The generalization of our theory for the case of the individually weighted entries of $\mathbf{A}_{j n}$ is not straightforward and is therefore beyond this paper's scope. The main difficulty is in deriving equations for the derivatives $\partial \varphi_{d} / \partial \sigma_{k}$. However, it still is possible to extend the theory to the more general case if the data misfit

$$
\varphi_{d}=\frac{1}{2} \sum_{j=1}^{N_{S}} \sum_{n=1}^{N_{T}} \operatorname{tr}\left[\overline{\widetilde{\mathbf{A}}}_{j n}^{T} \widetilde{\mathbf{A}}_{j n}\right]
$$

is written in terms of the weighted matrices

$$
\tilde{\mathbf{A}}_{j n}=\left(\begin{array}{ll}
w_{x x} A_{x x} & w_{x y} A_{x y} \\
w_{y x} A_{y x} & w_{y y} A_{y y}
\end{array}\right)_{j n},
$$

where the tilde means weighting by real-valued weights $w_{x x}, w_{x y}, w_{y x}, w_{y y}$. If we assume additionally that $w_{x y}=w_{y x}$, then it is easy to prove that $\widetilde{\mathbf{B}}^{T}=\widetilde{\mathbf{B}^{T}}$ and $\operatorname{tr}[\widetilde{\mathbf{B}} \mathbf{C}]=\operatorname{tr}[\mathbf{B} \widetilde{\mathbf{C}}]$ for any $\mathbf{B}$ and $\mathbf{C}$. These simple properties allow us to obtain the relevant alterations of equations presented in this paper and could be used to derive equation 14 for $\partial \varphi_{d} / \partial \sigma_{k}$.

It can be shown that equations $8,9,12,13$, and $\mathrm{C}-5$, respectively, alter to the following forms: 


$$
\begin{aligned}
\frac{\partial \varphi_{d}}{\partial \sigma_{k}}= & \operatorname{Re}\left\{\sum_{j=1}^{N_{S}} \sum_{n=1}^{N_{T}} \operatorname{tr}\left[\overline{\widetilde{\mathbf{A}}}_{j n}^{T} \frac{\partial \widetilde{\mathbf{Z}}_{j n}}{\partial \sigma_{k}}\right]\right\}, \quad(\mathrm{D}-3) \\
\frac{\partial \varphi_{d}}{\partial \sigma_{k}}= & \operatorname{Re}\left\{\sum _ { j = 1 } ^ { N _ { S } } \sum _ { n = 1 } ^ { N _ { T } } \operatorname { t r } \left[\overline { \hat { \mathbf { A } } } _ { j n } ^ { T } \left(\mathbf{E}_{j n, k}\right.\right.\right. \\
& \left.\left.\left.-\mathbf{Z}_{j n} \mathbf{H}_{j n, k}\right) \mathbf{H}_{j n}^{-1}\right]\right\}, \\
\mathbf{j}_{n}^{\mathrm{ext}}= & \sum_{j=1}^{N_{S}} \mathbf{p}^{T} \overline{\hat{\mathbf{A}}}_{j n} \mathbf{H}_{j n}^{-T} \delta\left(\mathbf{r}-\mathbf{r}_{j}\right), \\
\mathbf{h}_{n}^{\text {ext }}= & -\frac{1}{i \omega_{n} \mu} \sum_{j=1}^{N_{S}} \mathbf{p}^{T} \mathbf{Z}_{j n}^{T} \overline{\hat{\mathbf{A}}}_{j n} \mathbf{H}_{j n}^{-T} \delta\left(\mathbf{r}-\mathbf{r}_{j}\right),
\end{aligned}
$$

and

$$
\sum_{j=1}^{N_{S}} \operatorname{tr}\left[\overline{\hat{\mathbf{A}}}_{j n}^{T}\left(\mathbf{p} \mathbf{e}_{n k}\left(\mathbf{r}_{j}\right)-\mathbf{Z}_{j n} \mathbf{p} \mathbf{h}_{n k}\left(\mathbf{r}_{j}\right)\right) \mathbf{H}_{j n}^{-1}\right]=\int_{V_{k}} \operatorname{tr}\left[\mathbf{u}_{n}^{T} \mathbf{E}_{n}\right] d V,
$$

where

$$
\hat{\mathbf{A}}_{j n}=\left(\begin{array}{ll}
w_{x x}^{2} A_{x x} & w_{x y}^{2} A_{x y} \\
w_{y x}^{2} A_{y x} & w_{y y}^{2} A_{y y}
\end{array}\right)_{j n} .
$$

All other equations presented in the paper remain the same, including equation 14 .

\section{REFERENCES}

Abubakar, A., T. M. Habashy, V. L. Druskin, L. Knizhnerman, and D. Alumbaugh, 2008, 2.5D forward and inverse modeling for interpreting low-frequency electromagnetic measurements: Geophysics, 73, no. 4, F165F177.

Avdeev, D. B., 2005, Three-dimensional electromagnetic modelling and inversion from theory to application: Surveys in Geophysics, 26, 767-799.

Avdeev, D. B., A. V. Kuvshinov, O. V. Pankratov, and G. A. Newman, 1997 , High-performance three-dimensional electromagnetic modeling using modified Neumann series: Wide-band numerical solution and examples: Journal of Geomagnetism and Geoelectricity, 49, 1519-1539.

,- 2002 , Three-dimensional induction logging problems, Part I- An integral equation solution and model comparisons: Geophysics, 67,
413-426.

Avdeeva, A. D., and D. B. Avdeev, 2006, A limited-memory quasi-Newton inversion for 1D magnetotellurics: Geophysics, 71, no. 5, G191-G196.

Byrd, R. H., P. Lu, J. Nocedal, and C. Zhu, 1995, Alimited memory algorithm for bound constrained optimization: SIAM Journal on Scientific Computing, 16, 1190-1208.

Chen, J., D. W. Oldenburg, and E. Haber, 2005, Reciprocity in electromagnetics: Application to modeling marine magnetometric resistivity data: Physics of the Earth and Planetary Interiors, 150, 45-61.

Dorn, O., H. Bertete-Aguirre, J. G. Berryman, and G. C. Papanicolaou, 1999 , A nonlinear inversion method for 3D electromagnetic imaging using adjoint fields: Inverse Problems, 15, 1523-1558.

Farquharson, C. G., and D. W. Oldenburg, 1998, Non-linear inversion using general measures of data misfit and model structure: Geophysical Journal International, 134, 213-227.

Haber, E., 2005, Quasi-Newton methods for large-scale electromagnetic inverse problems: Inverse Problems, 21, 305-323.

Haber, E., U. Ascher, and D. Oldenburg, 2000, On optimization techniques for solving nonlinear inverse problems: Inverse Problems, 16, 1263-1280.

Mackie, R. L., W. Rodi, and M. D. Watts, 2001, 3D magnetotelluric inversion for resource exploration: 71st Annual International Meeting, SEG, Expanded Abstracts, 1501-1504.

Mackie, R. L., J. T. Smith, and T. R. Madden, 1994, Three-dimensional electromagnetic modeling using finite difference equations: The magnetotelluric example: Radio Science, 29, 923-935.

Mackie, R. L., M. D. Watts, and W. Rodi, 2007, Joint 3D inversion of marine CSEM and MT data: 77th Annual International Meeting, SEG, Expanded Abstracts, 574-578.

McGillivray, P. R., and D. W. Oldenburg, 1990, Methods for calculating Frechet derivatives and sensitivities for the non-linear inverse problems: Geophysics, 60, 899-911.

Miensopust, M., 2008, Comparison of the 3D forward responses: Presented at the MT 3D Inversion Workshop, DIAS, accessed 11 July 2008, http:// www.dias.ie/lang/en/cosmic/geo/mt_workshop2008.html.

Newman, G. A., and D. L. Alumbaugh, 2000, Three-dimensional magnetotelluric inversion using non-linear conjugate gradients: Geophysical Journal International, 140, 410-424.

Newman, G. A., and P. T. Boggs, 2004, Solution accelerators for large-scale three-dimensional electromagnetic inverse problem: Inverse Problems, 20, s151-s170.

Nocedal, J., and S. J. Wright, 1999, Numerical optimization: Springer.

Plessix, R.-E., and W. A. Mulder, 2008, Resistivity imaging with controlledsource electromagnetic data: Depth and data weighting: Inverse Problems, 24, 1-22.

Rodi, W., 1976, A technique for improving the accuracy of finite element solution for magnetotelluric data: Geophysical Journal of the Royal Astronomical Society, 44, 483-506.

Rodi, W., and R. L. Mackie, 2001, Nonlinear conjugate gradients algorithm for 2D magnetotelluric inversion: Geophysics, $\mathbf{6 6}, 174-187$.

Siripunvaraporn, W., G. Egbert, Y. Lenbury, and M. Uyeshima, 2005, Threedimensional magnetotelluric inversion: Data-space method: Physics of the Earth and Planetary Interiors, 150, 3-14.

Tikhonov, A. N., and V. Y. Arsenin, 1977, Solutions of ill-posed problems: John Wiley \& Sons, Inc.

Wannamaker, P. E., 1991, Advances in three-dimensional magnetotelluric modeling using integral equations: Geophysics, 56, 1716-1728.

Ward, S. H., and G. W. Hohmann, 1987, Electromagnetic theory for geophysical applications, in M. N. Nabighian, ed., Electromagnetic methods in applied geophysics: SEG, 131-311.

Weidelt, P., 1975, Inversion of two-dimensional conductivity structures: Physics of the Earth and Planetary Interiors, 10, 281-291.

Zhdanov, M. S., and E. Tolstaya, 2004, Minimum support nonlinear parameterization in the solution of a 3D magnetotelluric inverse problem: Inverse Problems, 20,937-952. 